Available online at GSC Online Press Directory

GSC Biological and Pharmaceutical Sciences

e-ISSN: 2581-3250, CODEN (USA): GBPSC2

Journal homepage: https://www.gsconlinepress.com/journals/gscbps

(RESEARCH ARTICLE)

\title{
Estimation of prothrombin time, activated partial thromboplastin time and thrombocytes among Sudanese patients with diabetes
}

\author{
Omer Ibrahim Abdallah Mohammed * \\ Omdurman Islamic University, Faculty of Medical Laboratory Sciences, Department of Hematology, Khartoum, Sudan.
}

Publication history: Received on 25 December 2019; revised on 12 January 2020; accepted on 13 January 2020

Article DOI: https://doi.org/10.30574/gscbps.2020.10.1.0250

\begin{abstract}
Diabetes mellitus is a common disease in Sudan, and it is a major cause of morbidity, several studies indicate that diabetes is a likely under reported as cause of death. This study aimed to evaluate of Prothrombin Time, Activated Partial Thromboplastin Time and Thrombocytes among Sudanese Patients with diabetes. This study includes 57 patients have diabetic and 20 persons as control. The blood coagulation markers, PT, INR and aPTT were measured by coagulation analyzer and thrombocytes count by hematological analyzer (Sysmex). Data analysis was performed using statistical package for social science (SPSS) software. Evaluation of patient's data was performed using the t-test and Pearson correlation test. Results with $\mathrm{p}$ value $<0.05$ were considered as statistically significant. This study concluded that patients with diabetes mellitus are more prone to develop hypercoagulation state, and it may be close to that diabetic patients are accelerated to develop coagulation impairment. Routine examinations of PT, INR, APTT and Thrombocyte count are important to assess coagulation impairment among diabetic patients in order to prevent any thromboembolic complication.
\end{abstract}

Keywords: PT; INR PTT; Platelet; Diabetes

\section{Introduction}

Diabetes mellitus is a common disease in Sudan, and it is a major cause of mortality, but several studies indicate that diabetes is likely underreported as cause of death. It has high prevalence therefore it is an important health problem. The problems of diabetic care include the lack of efficient diabetic care centers, lack of special trained personnel, poor compliance with therapy or diet [1]. Two types of diabetes mellitus are the most prevalent: type-1 diabetes is characterized by autoimmune destruction of pancreatic beta cells resulting in an absolute deficiency in insulin; and type 2 diabetes (T2DM), which corresponds to approximately $90 \%$ of cases of diabetes worldwide, is characterized by insulin resistance and/or reduced production of insulin [2]. Diabetes-related mortality is associated with thrombotic events, especially cardiovascular. In general, patients with diabetes present symptoms of hypercoagulability and hypo fibrinolysis. However, the mechanisms that trigger hemostatic abnormalities in diabetic patients are not clear [3]. A procoagulant condition is observed in diabetic patients, which ultimately contribute to cardiovascular events. The coagulation abnormalities observed in diabetic patients seem to be caused by the hyperglycemia, which also constitutes the distinguishing feature of this disease. These data are also supported by in vitro studies which demonstrate how glucose can directly determine alterations in the coagulation system. The abnormalities observed involve all stages of coagulation, affecting both thrombus formation and its inhibition, fibrinolysis, platelet and endothelial function [4]. Many clotting factors such as I, VII, IX, XII, Kallikrein and von Willebrand factor (VWF) are increased in diabetes. This hypercoagulability could be due to an imbalance between the endothelial surface and the blood clotting factors [5]. The purpose of this study is to estimate levels, PT, PTT, INR and number of thrombocytes among Sudanese patients suffering from diabetes.

\footnotetext{
${ }^{*}$ Corresponding author

E-mail address: omeribrahimabdallahmohammed@gmail.com
} 


\section{Material and methods}

This is observational, descriptive and cross sectional, conducted in Sudan-Khartoum and Algazeira states from May 2011 to July 2011.The subjects were selected from the coming patients to outpatients of emergency units. 57 persons with diabetes (type I\&II), with different age and sex.The inclusion criteria for the selection of cases were diagnosis of diabetes.The exclusion criteria Patients with disorder in which the proteins that control blood clotting become over active (disseminated intravascular coagulation), Patients with liver disease and Patients with Warfarin (Coumadin) use. Five $\mathrm{ml}$ of blood samples were drawn from everyone of study population, using standard venipuncture techniques. Sample was collected and then $2.5 \mathrm{ml}$ in trisodium citate centrifuged at $3000 \mathrm{rpm}$ for 15 minutes to obtain platelet poor plasma (PPP) and transparent plasma the separated plasma was analyzed to do PT, APTT or stored at 2-80c if not tested immediately [6] $2.5 \mathrm{~mL}$ of whole blood in K2 ethylene diamine tetra acetic acid. Sysmex KX-21 (hematology analyzer) to count thrombocyte [7]. Statistical analysis was performed using SPSS (SPSS, version16), data were expressed as mean and standard deviation $(\mathrm{M} \pm \mathrm{SD}$ ), the means were compared using independent T.test and Pearson's correlation analysis was used for correlation of parameters measured, P-value $<0.05$ was considered as statistically significant. This study was approved by faculty of medical laboratory sciences, Omdurman Islamic University, Khartoum, Sudan, and ethical clearance was obtained from ministry of health. All participant Patients was signed an informed consent before samples collection.

\section{Results}

The studied of total diabetic patients were 57 and 20 as control groups. They have been categorized into different ages whose frequencies are (20 - 74years) with different duration of disease and the majority of group between (41- 60 months). Fig I\&II. The results of PT in patient in mean was (16 seconds) and the mean of PT result in control was (14.6seconds), with P.value (0.003). The mean of INR results in patients was 1.3 and the mean of INR results in control were (1.1) with P. value (0.000). The results of PTT in patients in mean was (45 second) and the mean of PTT results in control were (34 seconds) with P.value (0.003). Table 1.

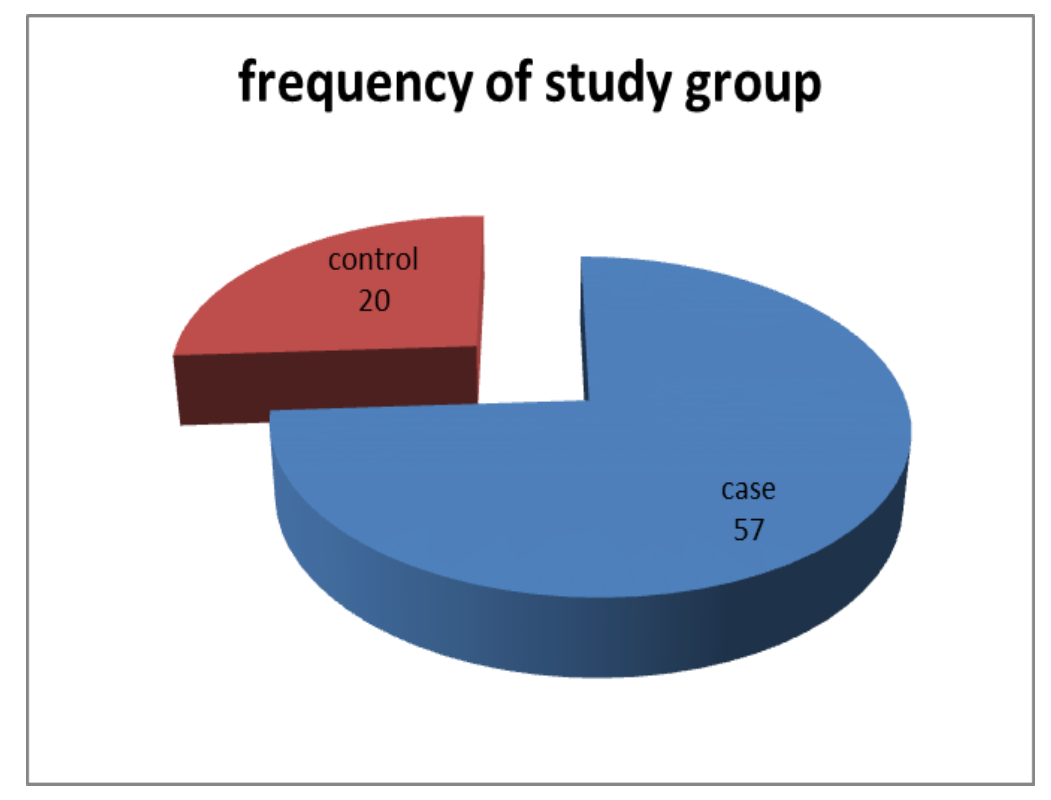

Figure 1 Show the frequency of study group 


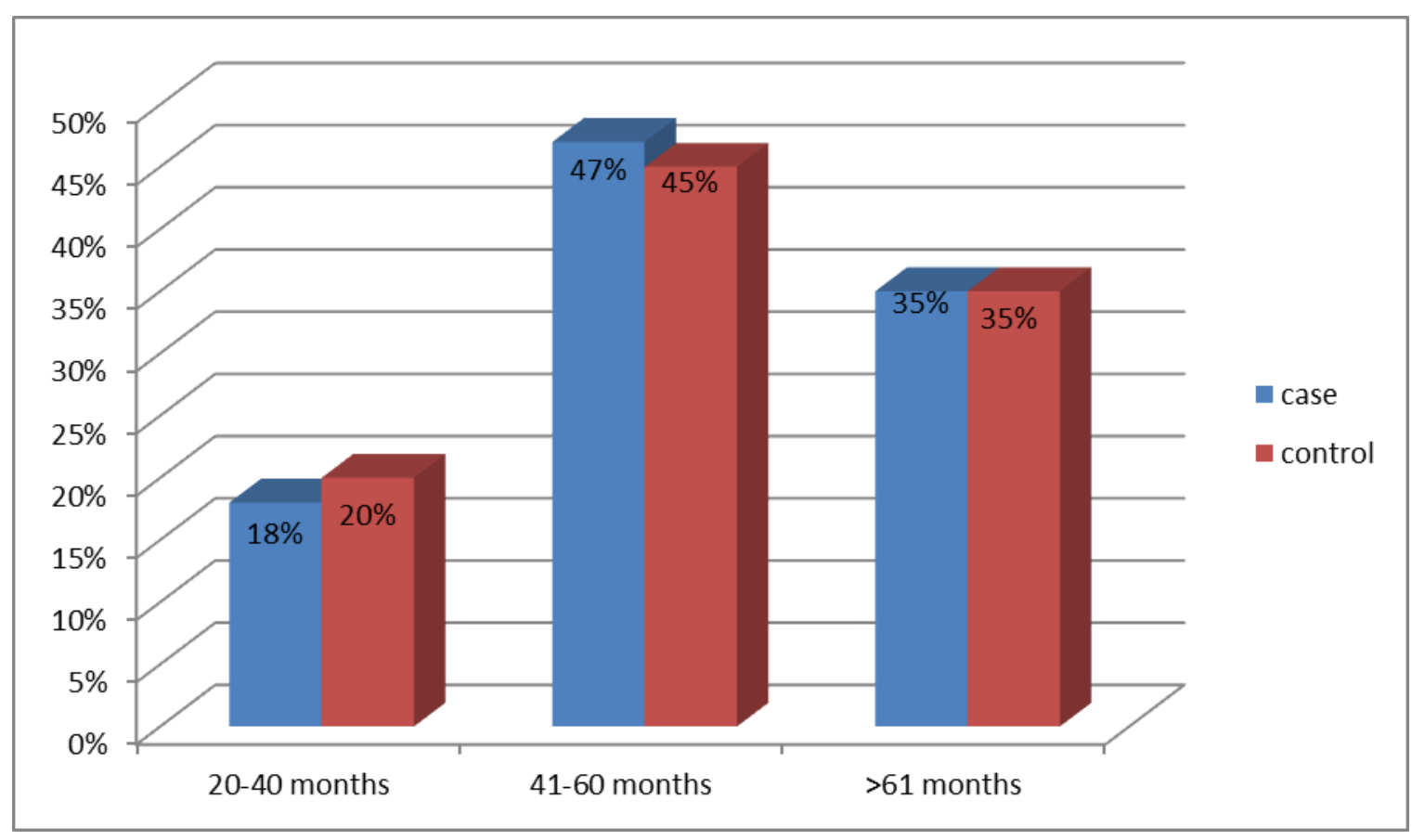

Figure 2 Comparison between case \& control in different duration

Table 1 Comparison of PT, INR, PTT and Platelets between case \& control

\begin{tabular}{llllll}
\hline Tests & Sample & N & Mean & Std. Deviation & P value \\
\hline Prothrombin time/ sec & case & 57 & 16.091 & 3.0626 & \\
& control & 20 & 14.570 & 1.3191 & 0.003 \\
International normalize ratio & case & 57 & 1.3214 & .27799 & \\
& control & 20 & 1.1375 & .12506 & 0.000 \\
Activated partial thromboplastin time/sec & case & 57 & 44.979 & 25.4686 & 0.003 \\
& control & 20 & 34.320 & 3.6222 & \\
Platelet count cell $\times 103 / \mathrm{L}$ & case & 57 & 194.54 & 79.31021 & 0.001 \\
& control & 20 & 262.95 & 70.72815 &
\end{tabular}

\section{Discussion}

The current study was conducted to estimate coagulation tests among Diabetic patients and normal healthy individuals. Diabetes is characterized by heavy risk of atherothrombotic complications affecting the cerebral, coronary and peripheral arterial trees. PT is an indicator of defects extrinsic and common pathway while APTT indicates in intrinsic and common pathway. This may account for abnormalities in hemostasis. Platelet abnormalities and dysfunction in coagulation cascade can elevated atherogenesis in diabetic patients. Insulin resistance (IR) is a uniform finding in type 2 diabetes, as are abnormalities in the macrovascular and microvascular circulations [8]. Our study shows that significantly increased values APTT and PT and INR among diabetic patient. Similar findings were observed in oltani et al. [9]. Prolonging of time period of coagulation tests and involvement of intrinsic coagulation pathway has also been confirmed by Soltani et al, [10]. But also, some studies found no significant changes in coagulation studies among diabetic patients $[11,12,13]$. Whereas few studies found lower of coagulation tests in diabetic patients [14,8]. Again, in the present study, we found the platelets signiant lower when was compare to our case control. Similar findings were 
observed in study conducted by Hekimsoy et al. [15]. It is however in contrast with the findings of a studies conducted by Thomas et al. [16], and Akinsegun et al [17]. Also, another study conducted by Chen et al found Thrombocyte, was not significantly different between diabetic patients and non-diabetic population [18]. Numerous studies have shown that coagulation abnormalities occur in the course of diabetes mellitus, resulting in a state of thrombophilia [4]. In Sudan there are many studies conducted about evaluation of coagulation impairment and mechanism of changing about liver disease and pregnancy, and few studies among diabetic, the affect among patient with liver disease were clearly significant [19,20,21], but in diabetes some studies found significant and other not, till now the hemostatic impairment it does not clear till now. The major limitations of our study are the sample size and duration of the study. Also, the study it should including more advance tests like thrombin generation assay and quantitative measuring to all coagulation factors.

\section{Conclusion}

Our study it may be concluded that patients with diabetes mellitus are more prone to develop hypercoagulation state, and it may be close to that diabetic patients are accelerated to develop coagulation impairment. Routine examinations of PT, INR, APTT and Thrombocyte count are important to assess coagulation impairment among diabetic patients in order to prevent any thromboembolic complication.

\section{Compliance with ethical standards}

\section{Acknowledgments}

The authors express gratitude to the Omdurman Islamic university, Faculty of Medical Laboratory Sciences, Department of Hematology, Also we are grateful thank all the patients and healthy who agreed to participate in this study.

\section{Disclosure of conflict of interest}

The authors declare no conflicts of interest.

\section{References}

[1] Rashid, Sarra Izzeldin, Nuha Mohamed A. Agabna and Asaad MA Babker. "Evaluation of Insulin Prescriptions in Type 2 Diabetic Patients in Jabir Aboeliz Health Center. Scholars Academic Journal of Pharmacy, 6(2), 67-70.

[2] Zimmet P, Alberti KG and Shaw J. (2001). Global and societal implications of the diabetes epidemic. Nature, 414(6865), 782-7.

[3] Soares, Anna L, Sousa, Marinez dO, Fernandes, Ana PSM, Carvalho and Maria dG. (2010). Hemostatic changes in patients with type 2 diabetes mellitus. Revista Brasileira de Hematologia e Hemoterapia, 32(6), 482-488.

[4] Ceriello A. (1993). Coagulation activation in diabetes mellitus: the role of hyperglycaemia and therapeutic prospects. Diabetologia 36, 1119-1125.

[5] Bashir BA and Ali MS. (2018). Hemostatic state augmented with platelet indices among Sudanese diabetic septic foot. BMC hematology, 18, 11.

[6] Ali NM, Gameel FE, Elsayid M and Babker AM. (2016). Alterations in D-Dimer, Prothrombin Time and Activated Partial Thromboplastin Time as Thrombogenesis Activity Markers in Patients with Acute Myocardial Infarction. Open Journal of Blood Diseases, 11, 6(01), 1.

[7] Agabeldour AA, Khalafallah TO and AbdAllah AM. (2015). Hematological changes among Sudanese petroleum workers with a broad range of benzene exposure: Sch. J. App. Med. Sci., 3(8D), 3054-6.

[8] Ankalayya B, HS Sodhi, Sudhir Modala and Manisha Baghel. (2016). A Comparative study of coagulation time in type 2 diabetes mellitus and healthy individuals. International Journal of Contemporary Medical Research, 3(11), 3170-3171.

[9] Soltani MM, Dayer MR, Zahed AS, Bahar HA and Nasirbagheban Z. (2012). The Buffering role of HDL in balancing the effects of hypercoagulable state in type 2 Diabetes. J Appl Sci., 12(8), 745-52. 
[10] Mard-Soltani M., MR Dayer, M Taheri-Shoshi, G Ataie, AA Moazedi and AH Shamshirgar-Zadeh. (2011). Cholesterol and lipoproteins have an important role in homeostasis of coagulation factors in type 2 diabetes mellitus. Clin. Biochem., 44, S55-S59.

[11] Bae SH, Lee J, Roh KH and Kim J. (2003). Platelet activation in patient with diabetic retinopathy. Korean J Opthalmol, 17, 140-4.

[12] Hamid RDMN. (2017). Estimation of Prothrombin Time, Activated Partial Thromboplastin Time and platelets count in Diabetes Type II patients in Khartoum State (Doctoral dissertation, Sudan University of Science \& Technology).

[13] Matcas HA. (2009) Study on the anomalies of hemostasis on a group of dislipoproteinemia patients [master thesis]. [Craiova]: University of Medicine and Pharmacy Craiova, 139.

[14] Karim F, Akter QS, Jahan S, Khanom A, Haque S, Yeasmin T, Siddika T and Sinha S. (2015). Coagulation Impairment in type 2 diabetes mellitus. Journal of Bangladesh Society of Physiologist, 21, 10(1), 26-9.

[15] Hekimsoy Z, Payzinb B, Ornek T and Kandogan G. (2004). Mean platelet volume in Type 2 diabetic patients. J Diabetes Complications, 18(3), 173-176.

[16] Thomas AK, Udaya KM, Suraksha BR, Thej MJ, Madheri R, Harendra KML and Venkataswamy L. (2012). Mean platelet volume in type 2 diabetes mellitus. J lab physicians, 4(1), 5-9.

[17] Akinsegun A, Akinola Olusola D, Sarah JO, Olajumoke O, Adewumi A, Majeed O, Anthonia O, Ebele U, Olaitan O, Olanrewaju A and Kingsley A. (2014). Mean platelet volume and platelet counts in type 2 diabetes: mellitus on treatment and non-diabetic mellitus controls in Lagos, Nigeria. Pan Afr Med J. 12, 18, 42.

[18] Chen X, Fang L, Lin H, Shen P, Zhang T, Li H, Li X, Yu M, Xu C, Zhang J, Lu F, DU X, Hu R and Zhong J. The Relationship between Type 2 Diabetes and Platelet Indicators. Iran J Public Health. 2017 Sep, 46(9), 1211-1216.

[19] Babker AM and Gameel FE. (2015). Molecular Characterization of Prothrombin G20210A gene Mutations In pregnant Sudanese women with spontaneous recurrent abortions. Rawal Medical Journal, 40(2):207-9.

[20] Mohamed AO, Hamza KM and Babker AM. (2016). Physiological changes in some hematological and coagulation profile among Sudanese healthy pregnant women. Int J Med Sci Public Health. 1, 5(3), 525-8.

[21] Mustafa, ME, Mansoor MM, Mohammed A and Babker AA. (2015). Evaluation of Platelets Count and Coagulation Parameters among Patients with Liver Disease. World Journal of Pharmaceutical Research, 4, 360-368.

\section{How to cite this article}

Omer Ibrahim AM. (2020). Estimation of prothrombin time, activated partial thromboplastin time and thrombocytes among Sudanese patients with diabetes. GSC Biological and Pharmaceutical Sciences, 10(1), 34-38. 